\title{
Metabolic Effects of Social Isolation in Adult C57BL/6 Mice
}

\author{
Meng Sun, ${ }^{1}$ Eugene Y. Choi, ${ }^{1}$ Daniel J. Magee, ${ }^{1}$ Colin W. Stets, ${ }^{1}$ \\ Matthew J. During, ${ }^{1,2}$ and En-Ju D. Lin ${ }^{1}$ \\ ${ }^{1}$ Department of Molecular Virology, Immunology and Medical Genetics and the Comprehensive Cancer Center, \\ The Ohio State University, 912 Biomedical Research Tower, 460 West 12th Avenue, Columbus, OH 43210, USA \\ ${ }^{2}$ Functional Genomics and Translational Neuroscience Lab, Department of Molecular Medicine and Pathology, \\ University of Auckland, Auckland 1142, New Zealand
}

Correspondence should be addressed to En-Ju D. Lin; en-ju.lin@osumc.edu

Received 27 August 2014; Accepted 23 October 2014; Published 26 November 2014

Academic Editor: Isabel C. F. R. Ferreira

Copyright (C) 2014 Meng Sun et al. This is an open access article distributed under the Creative Commons Attribution License, which permits unrestricted use, distribution, and reproduction in any medium, provided the original work is properly cited.

\begin{abstract}
Obesity and metabolic dysfunction are risk factors for a number of chronic diseases, such as type 2 diabetes, hypertension, heart disease, stroke, and certain forms of cancers. Both animal studies and human population-based and clinical studies have suggested that chronic stress is a risk factor for metabolic disorders. A good social support system is known to exert positive effects on the mental and physical well-being of an individual. On the other hand, long-term deprivation of social contacts may represent a stressful condition that has negative effects on health. In the present study, we investigated the effects of chronic social isolation on metabolic parameters in adult C57BL/6 mice. We found that individually housed mice had increased adipose mass compared to group-housed mice, despite comparable body weight. The mechanism for the expansion of white adipose tissue mass was depotspecific. Notably, food intake was reduced in the social isolated animals, which occurred around the light-dark phase transition periods. Similarly, reductions in heat generated and the respiratory exchange ratio were observed during the light-dark transitions. These phase-specific changes due to long-term social isolation have not been reported previously. Our study shows social isolation contributes to increased adiposity and altered metabolic functions.
\end{abstract}

\section{Introduction}

Social isolation (SI) has been shown to induce an increase in emotionality and hypothalamic-pituitary-adrenal axis reactivity in mice $[1,2]$. Long-term individual housing has been used as a chronic stress model in the study of psychiatric disorders [3-5] and, to a lesser extent, metabolic disorders [6]. Given the association between stress and obesity $[7,8]$, efforts have been made in developing and characterizing animal stress models for the study of metabolic dysfunctions. These models include unpredictable chronic stress model, social defeat model, and the use of various chronic stressors [9-11].

The hypothalamo-pituitary-adrenal (HPA) axis and the sympathetic nervous system (SNS) are the main effector pathways of the stress system and both affects energy metabolism. During acute stress, activation of the SNS triggers the release of epinephrine, which mediates a number of physiological changes to prepare the body for "fight or flight" response.
These changes include increased heart rate, increased blood flow to vital organs, and mobilization of energy source by enhanced lipolysis and hepatic gluconeogenesis [12]. At the same time, corticotropin-releasing hormone $(\mathrm{CRH})$ is released from the paraventricular nucleus (PVN) of the hypothalamus. CRH causes an immediate suppression of food intake through inhibiting the appetite-stimulating neuropeptide Y (NPY)/agouti-related peptide (AGRP) neurons in the arcuate nucleus (ARC), thereby diverting the body's resources to deal with the stressor [13-16]. CRH also induces the release of adrenocorticotropic hormone (ACTH) from the pituitary. The circulating ACTH in turn stimulates the adrenal cortex to secrete glucocorticoids, which regulate metabolism and food intake through a number of mechanisms $[17,18]$. In addition to stimulating feeding responses by inhibiting CRH release and increasing NPY release in the hypothalamus [19], cortisol also upregulates genes encoding lipoprotein receptors and enzymes of glucose, lipid, and amino acid metabolism (see [18]). Moreover, stress-induced 
elevations in glucocorticoids secretion appear to intensify emotions and motivation $[20,21]$ and promote the intake of palatable foods $[22,23]$, thus contributing to the development of "comfort eating" [24, 25]. Ethologically, the appetitestimulatory effect of the glucocorticoids serves to replenish the energy used when the stressful event subsides.

In a normal stress response, the activation of both the HPA axis and the SNS is of limited duration and would return to baseline when homeostasis is achieved. However, in chronic stress, prolonged activation of the stress system can lead to a disruption of homeostasis. Based on the current understanding of the stress response, it is reasonable to postulate that chronic stress lead to chronic hypercortisolemia, which promotes feeding and hence excessive weight gain. However, both human and animal studies showed that chronic stress could lead to either increase or decrease of food intake and body weight [26-30]. The reason for the bidirectional response to stress is not fully understood and is likely to involve many factors $[31,32]$. One explanation is the balance between an increase in $\beta$-adrenergic activation, the body's main fat-burning mechanism (leading to weight loss), and the increased intake of sugar- and fat-rich comfort foods (resulting in weight gain) $[25,33,34]$. The observation that those who are initially overweight are more inclined to increase body weight when stressed whereas those who are of normal- or underweight do not led Dallman to propose that difference in metabolic outcomes might be the results of higher insulin concentration in people with higher body mass index [24]. More recently, others have suggested that individual differences in ghrelin level and response may contribute to the variability in stress-induced appetite [35].

Animal models are useful for understanding the complex interaction between stress and metabolic processes. Similar to human observation, these animal models have produced variable and even opposite phenotypes of food intake, body weight gain, and adiposity. The variable metabolic phenotypes observed in the different studies have been attributed to the types of stressors, diets, protocol durations, strains of animals, and stress intensities. The variability highlights the importance of a thorough characterization of different stress models. SI is a simple model with minimal handling and technical requirement. However, only a handful of studies directly investigated the effect of SI on body weight regulation and metabolic functions. Conflicting results were reported for the effects of SI, whereas some observed an increase $[36,37]$, others observed a decrease in body weight gain [3842]. In this study, we assessed the effect of SI on body weight gain and adiposity in adult C57BL/6J mice after two months of single housing. We also monitored the food intake, activity, heat production, and respiratory exchange ratio (RER) of the mice using a metabolic chamber.

\section{Materials and Methods}

2.1. Animals. Twelve-week-old male C57BL/6 mice (Charles River Laboratories, Wilmington, MA) were housed in groups of four (control; $n=20$ ) or single-housed (SI; $n=16$ ) in standard cage $(31 \mathrm{~cm} \times 17 \mathrm{~cm} \times 14 \mathrm{~cm})$ for 8 weeks. All mice were kept under a $12 \mathrm{~h}$ light/dark cycle (lights on at
$0600 \mathrm{hr}$ ), with free access to water and food (Diet 7012, Harlan Laboratories). All use of animals was approved by the Ohio State University Animal Care and Use Committee and was in accordance with the NIH guidelines.

2.2. Metabolic Chamber Analysis. Following eight weeks of SI (or control housing), a subset of mice ( $n=4$ per group) was individually placed in the chambers of the Oxymax Lab Animal Monitoring system (Columbus Instruments, $\mathrm{OH}$ ). Following 2 days of acclimatization, food intake, activity, heat generated, and respiratory exchange ratio (RER) were recorded in day 3 .

2.3. Tissue Collection. Eight weeks after the start of isolation, mice were culled by decapitation under isoflurane anesthesia. Trunk blood was collected and serum was isolated by centrifugation and stored at $-20^{\circ} \mathrm{C}$ until assayed. Brown adipose tissue (BAT), inguinal (WATi), retroperitoneal (WATr), and epididymal (WATe) white adipose tissue were dissected and weighed to determine body composition and adiposity. WAT depots were collected from one side only, immersed in $4 \%$ paraformaldehyde and paraffin-embedded for subsequent morphological analysis. For tissue weight measurements, the subset of mice that underwent metabolic chamber analysis was not included as mice were euthanized by a later time point. One SI mouse was excluded because of a large mass found in the abdominal cavity. Tissue were dissected and weighed from 14 control mice instead of 16 due to time constraint.

2.4. WAT Staining and Adipocyte Size Analysis. Paraffinembedded WAT was cut into $20 \mu \mathrm{m}$ sections using a cryostat and mounted on slides. Sections were stained with hematoxylin and eosin (H\&E) to examine the degree of adipocyte hypertrophy. Adipocyte diameter was measured using the Quick Measure Circle Command in Stereo Investigator 7 (MBF Bioscience, Willeston, VT) using a 10x objective. Software generated $150 \mu \mathrm{m}$ grids were drawn over the entire section; the cells within every other grid were circled to estimate diameter of the circle, excluding any incomplete cells. At least 10 cells per section were measured and then averaged.

2.5. Serum Biomarker Analysis by ELISA. Serum was analyzed for leptin, leptin receptor, adiponectin, and insulinlike growth factor-1 (IGF-1) using DuoSet ELISA Development Systems (R\&D Systems, Minneapolis, MN) according to manufacturer's instruction. Serum corticosterone level was determined using Enzyme Immunoassay Kit at 1:200 dilution according to the manufacturer's instruction (Assay Designs, Inc., Ann Arbor, MI).

2.6. Statistical Analysis. Statistical analysis was performed using JMP software (SAS Institute Inc., Cary, NC). For food intake, activity, heat generated, and RER over time, we determined the overall significance by repeated measure ANOVA while pair-wise comparison of individual timepoints was assessed using Student's $t$-test. All other results 


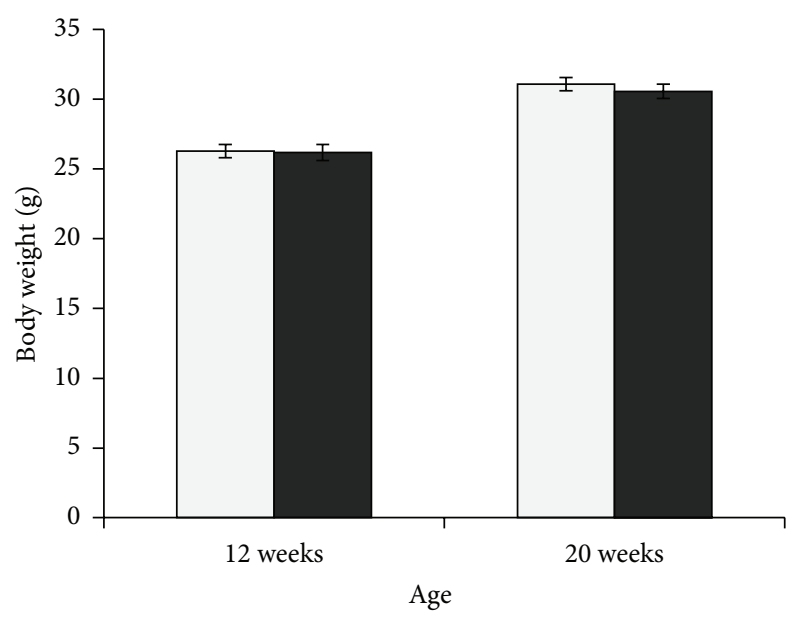

$\square$ Isolation
Control

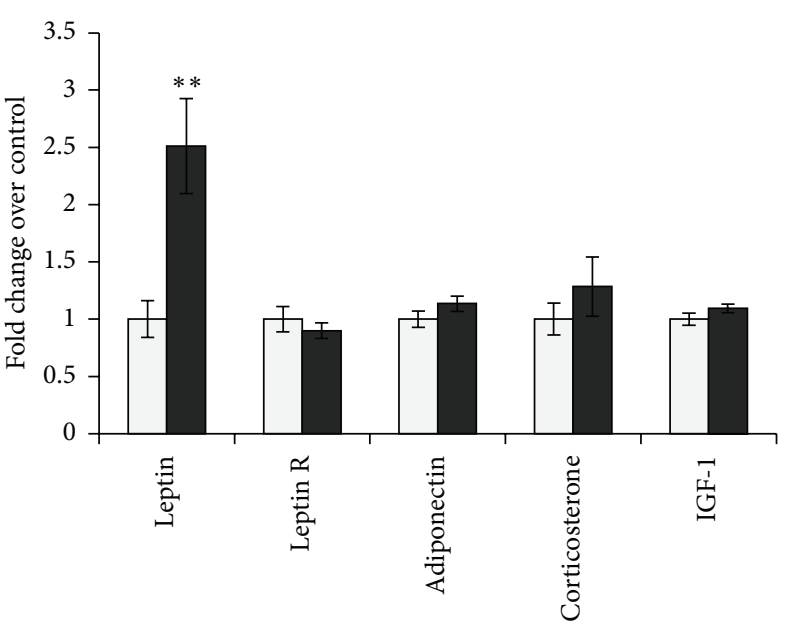

Control

Isolation

(a)

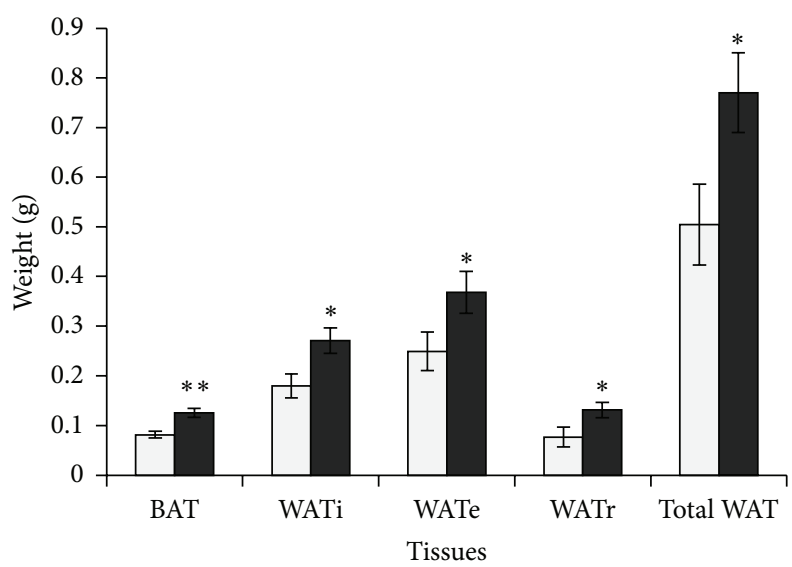

$\square$ Control

Isolation

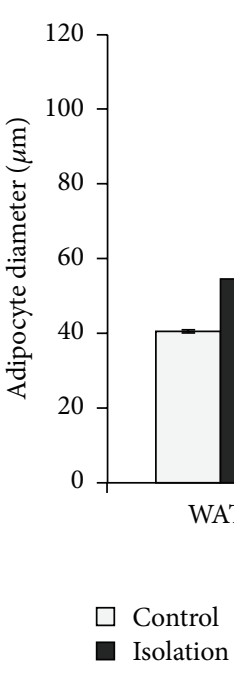

(b)

(d)

FIGURE 1: (a) Body weight at the start of the experiment (12 weeks) and following 2 months of differential housing conditions at 20 weeks was comparable between the groups ( $n=11$ for SI group, $n=14$ for group-housed control). (b) Serum biomarker profile after 2 months of social isolation showed robust increase in leptin level ( $n=7-20$ per group). (c) Interscapular BAT and WAT depots (from one side) were all increased by SI (SI, $n=11$, control, $n=14)$. (d) Adipocyte diameters were greater in WATi and WATr but not WATe in the SI mice $(n=3-5$ mice per group, each mouse with over 10 measurements). Data shown are mean \pm S.E.M. ${ }^{*} P<0.05 .{ }^{* *} P<0.01$.

were analyzed by Student's $t$-test for significant differences between the control and the isolation groups. Statistical significance was set at $P<0.05$. All data are presented as means \pm standard error of the mean (S.E.M).

\section{Results}

3.1. SI Increased Adiposity without Increase in Body Weight. Eight weeks of social isolation significantly increased adiposity without significant body weight gain (Figures 1(a) and 1(c)). Brown adipose tissue (BAT) weight was increased by 1.54 -fold $(P<0.01)$. WATi, WATe, and WATr weights showed increases of 1.51-fold $(P<0.05)$, 1.48-fold $(P<0.05)$, and 1.72 -fold $(P<0.05)$, respectively. The combined WAT from the three depots was increased by 1.53 -fold $(P<0.05)$ in the single-housed mice. Consistent with the increase in adiposity, the SI group showed marked increase in serum leptin level $(P<0.01$, Figure 1(b)). In contrast, levels of serum adiponectin, corticosterone, IGF-1, and leptin receptor were comparable between isolated and group-housed mice.

\subsection{SI Increased WAT Tissue Mass via Distinct Mechanisms} in Different Depots. We compared the adipocyte diameters in the two groups to examine whether the increase in 


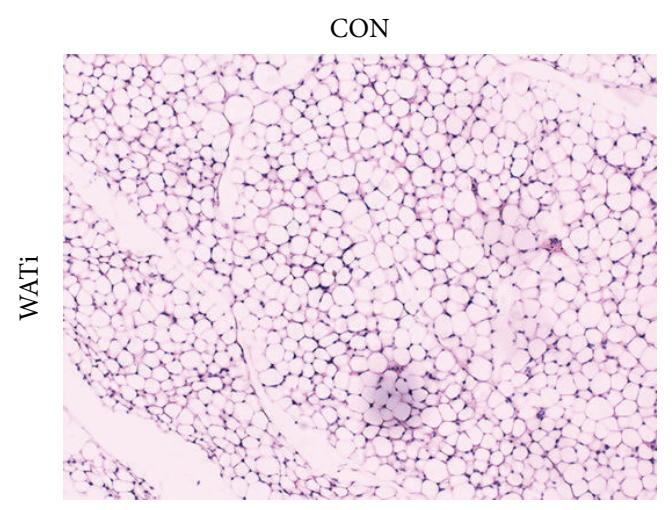

(a)

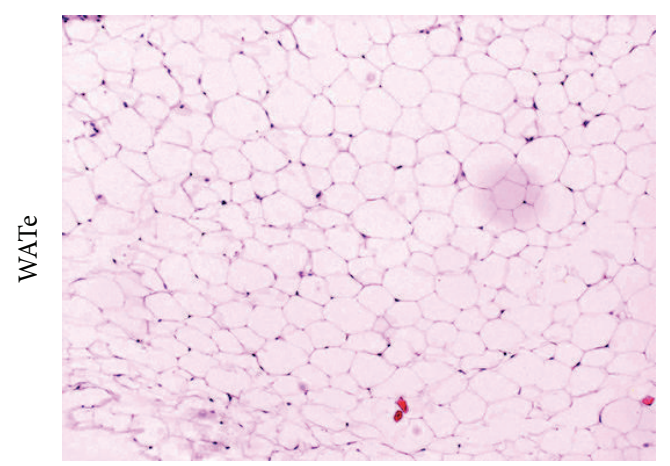

(b)

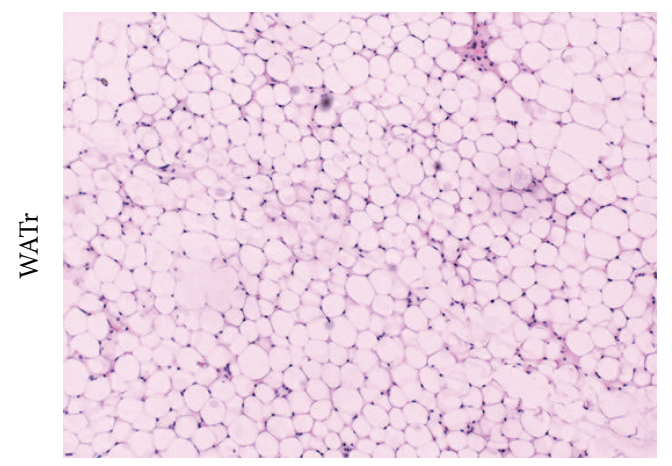

(c)

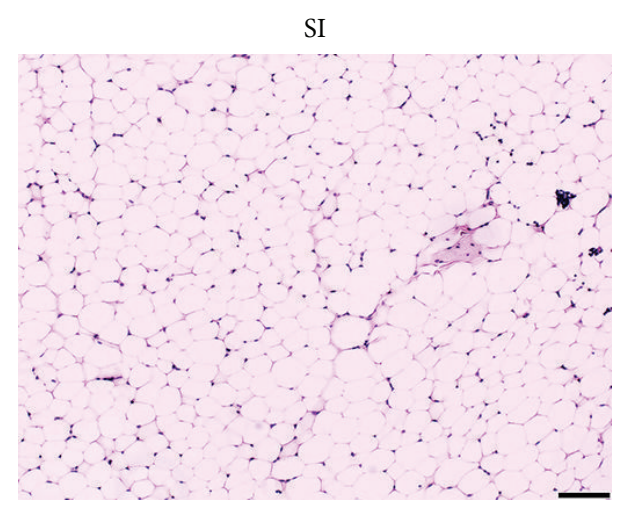

(d)

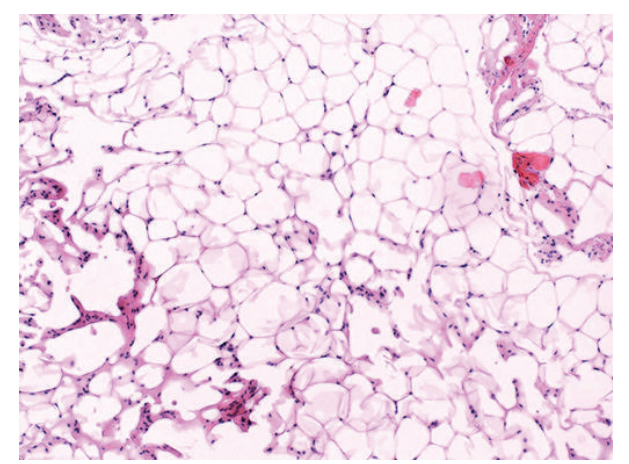

(e)

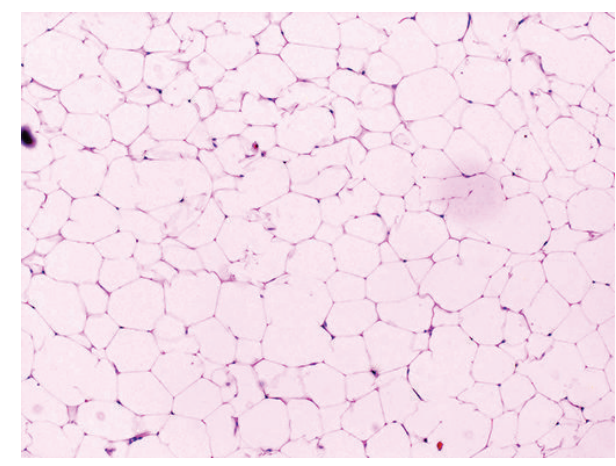

(f)

FIGURE 2: Representative sections of H\&E stained adipose tissue showing WATi and WATr adipocytes were enlarged in SI mice ((d) and (f), resp.) compared to control C57BL/6 mice ((a) and (c), resp.). In contrast, WATe adipocytes were comparable in size between the two groups ((b) and (e)). Scale bar $=100 \mu \mathrm{m}$.

WAT tissue mass was due to increased adipocyte number (hyperplasia) or increase in adipocyte size (hypertrophy). Interestingly, the effects were depot-specific. In the SI group, the adipocytes were significantly larger for WATi and WATr, by $1.3-(P<0.05)$ and $2.1-(P<0.01)$ fold, respectively. In contrast, the cell sizes of WATe were comparable between the two groups (Figure 1(d)). Figure 2 shows representative sections of the different WAT depots in the control and isolated mice.

3.3. SI Reduced Food Intake and RER at the Light-Dark Transition Periods. Daily food intake was reduced in the SI group $(P<0.01$, Figure $3(\mathrm{~b}))$. Repeated measure ANOVA revealed a significant effect over time $(P<0.0001)$, although the interaction effect between time and housing condition was not significant $(P=0.202)$. A group effect was detected $(P<0.01)$. Notably, the difference in food intake between the two groups was most dramatic at the transition periods between light and dark phases (Figure 3(a)).

A similar observation was made for energy expenditure and RER over time (Figures 3(e) and 3(g)), with differences detected at the transition periods between light and dark phases. Interaction effects were detected between time and housing condition for both energy expenditure $(P<0.05)$ and RER $(P<0.05)$, indicating that SI exerted differential effects depending on the time of day. The reduction in energy expenditure in the isolated mice was subtle and did 


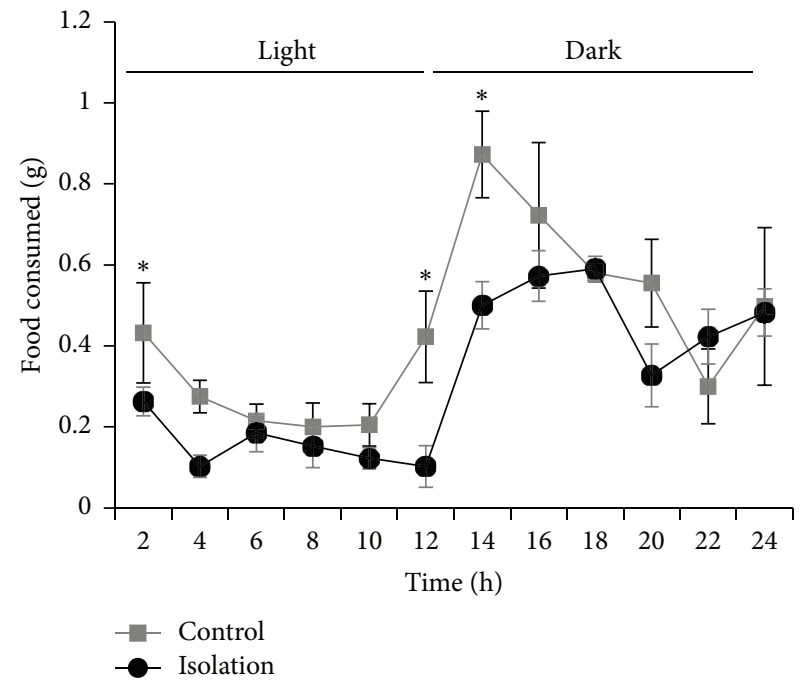

(a)

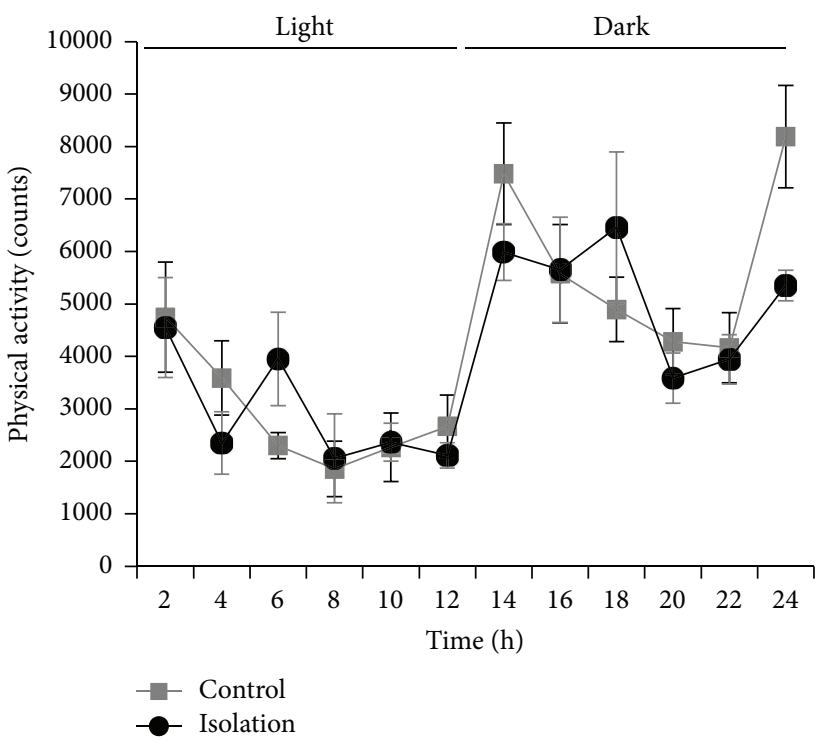

(c)

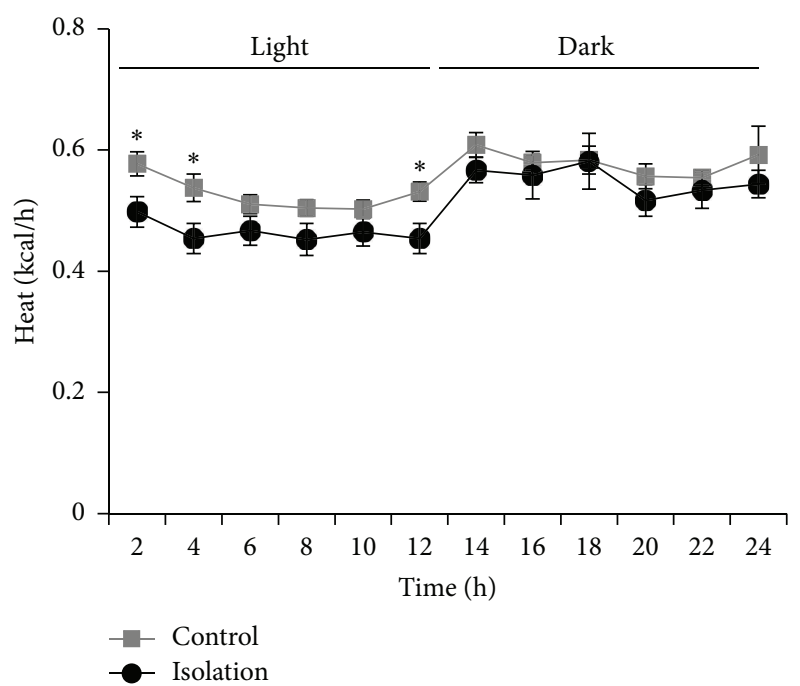

(e)

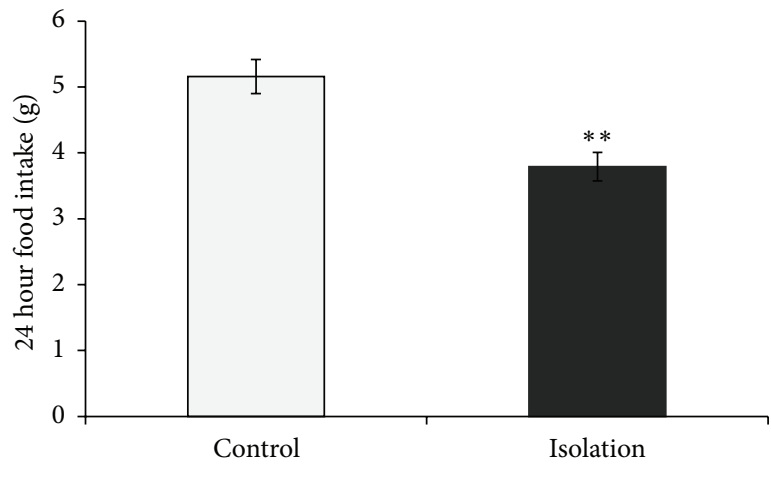

(b)

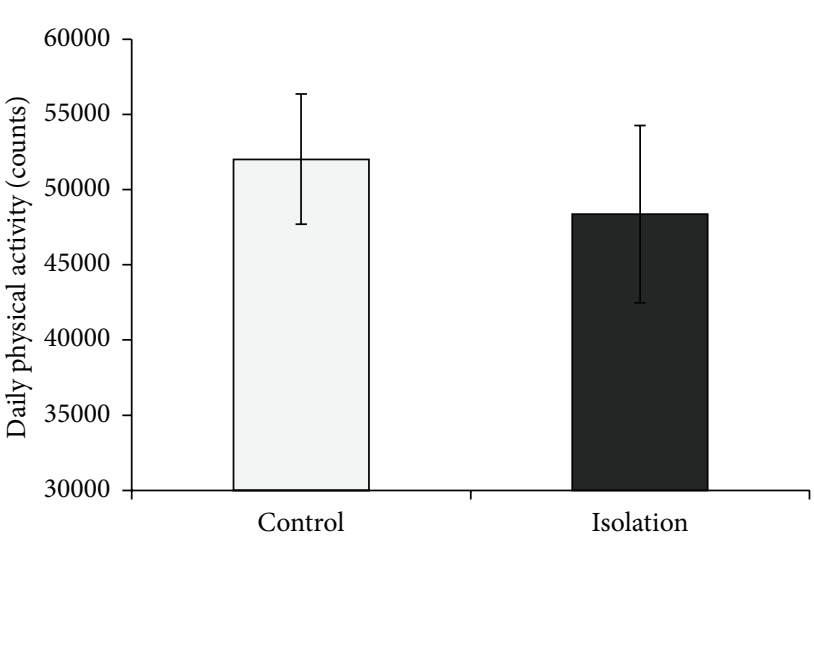

(d)

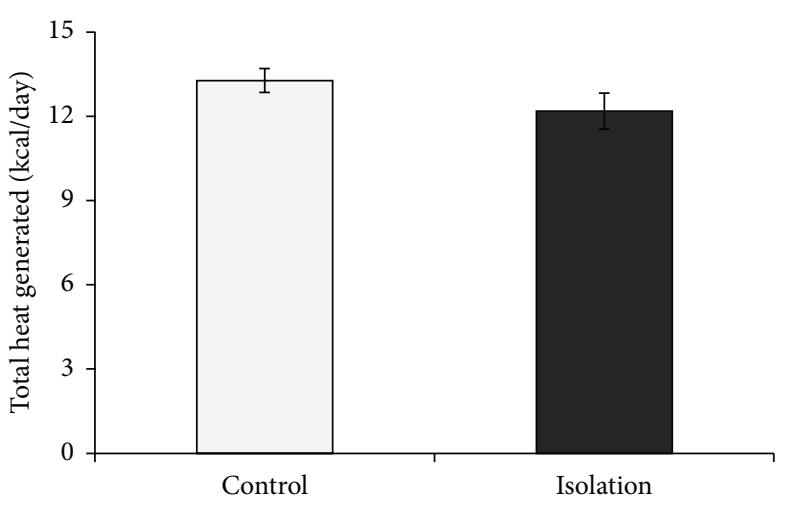

FIgUre 3: Continued. 


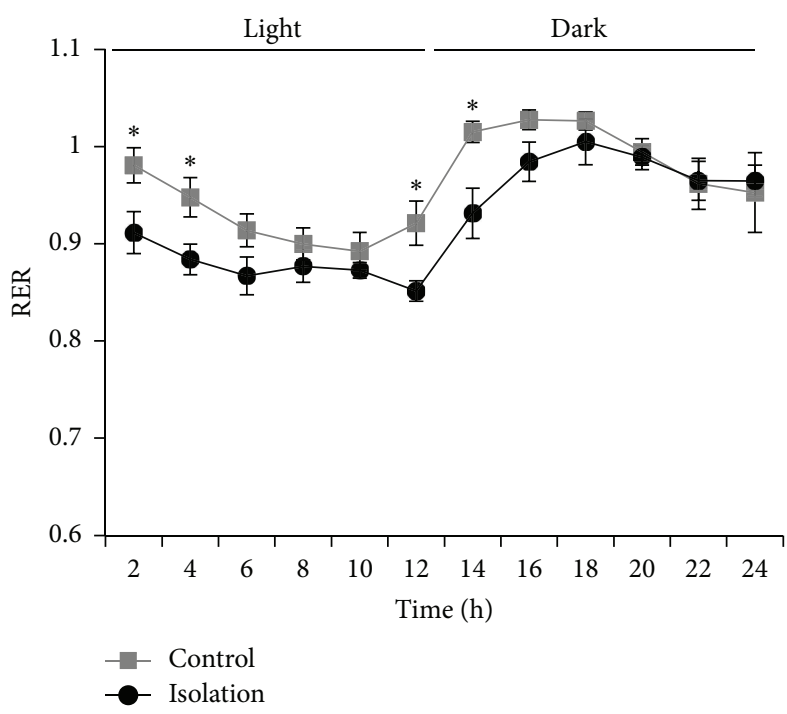

(g)

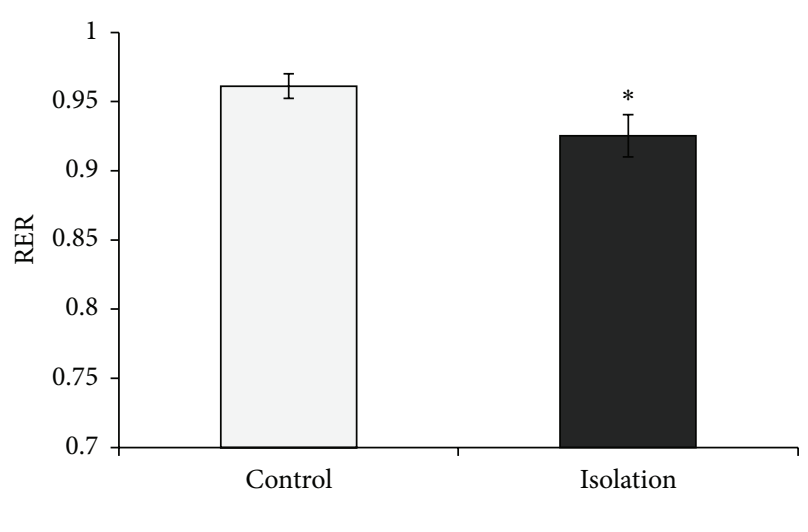

(h)

FIGURE 3: Effects of SI on food intake ((a) and (b)), physical activity ((c) and (d)), energy expenditure ((e) and (f)), and respiratory exchange ratio $((\mathrm{g})$ and $(\mathrm{h}))(n=4$ per group). Social isolation caused a reduction in food intake (a) and RER (g) and a subtle effect on energy expenditure (heat (e)) around light-dark transition periods. Data shown are mean \pm S.E.M. ${ }^{*} P<0.05 .{ }^{* *} P<0.01$.

not reach statistical significance when total daily energy expenditure was compared (Figure 3(f)). In contrast, the RER was significantly lower in the SI group $(P<0.05$; Figure $3(\mathrm{~h}))$, suggesting a shift from carbohydrate to lipid oxidation. Daily physical activity was unaffected by SI (Figures 3(c) and 3(d)).

\section{Discussion}

Animal models of chronic stress are important tools for the study of the pathophysiology and molecular mediators of stress-related disorders such as anxiety and metabolic syndromes. These animal models are also needed for the verification of potential treatments. Our study shows that two months of SI can produce profound effect on body composition despite minimal effect on body weight. In line with this observation, a recent report also showed that 4week-old C56BL/6J mice individually housed for 13 weeks had increased body weight and visceral fat compared to group-housed mice, evident after 9 weeks of SI [37]. Here we observed an increase in adiposity prior to any significant body weight gain. Although it remains possible that longer duration of social isolation may eventually lead to significant increases in body weight gain, our study shows that there was a change in body composition and a preferential accumulation of adipose tissues even before any observable body weight gain.

Consistent with the increased adipose mass, the SI mice exhibited a marked increase in serum leptin. Interestingly, we did not observe changes in serum adiponectin, which often change in opposite direction to serum leptin and are known to decrease under obese conditions [43-45]. A previous study by Sakakibara et al. found decreased plasma adiponectin after 13 weeks of social isolation, correlating to an increased body weight gain of the isolated mice, but intriguingly plasma leptin was unchanged [37]. In contrast, our finding is similar to that reported by Nonogaki et al., in which the socially isolated mice exhibited increased WATe weight, BAT weight, and plasma leptin, but no change in plasma adiponectin level [6]. In human, obesity can lead to marked changes in the growth hormone (GH) - IGF-1 axis [46]. Both increases and decreases in circulating IGF-1 have been reported in obese subjects [47-50]. Chronic stress can also lead to decreased IGF-1 levels $[51,52]$. In the present study, we did not observe any significant change in serum IGF-1 level. This may reflect the lack of body weight change in the SI mice and indicates that chronic social isolation stress exerts a greater effect on adiposity with little impact on the somatotropic axis.

Serum corticosterone was unchanged in the SI mice after 8 weeks of individual housing, consistent with a recent study that showed comparable basal corticosterone level in adult C57BL/6J male mice after 4 weeks of social isolation [1]. This was likely due to an adaptation to the chronic stress. A return to comparable baseline corticosterone following chronic psychosocial stress has been reported by the others using the chronic subordinate colony housing paradigm in mice $[53,54]$. Similarly, using a social overcrowding stress model, we observed an increase in serum corticosterone in the first three weeks and then returned to baseline by 8 weeks [55]. It is interesting to note that although baseline corticosterone might have returned to normal, these mice could exhibit enhanced stress-induced corticosterone release as shown in a previous study [1].

Although all WAT depots were enlarged, only WATi and WATr showed an increase in adipocyte size, indicating that the enlargement of these depots were at least in part due to adipocyte hypertrophy. In contrast, WATe adipocyte sizes were comparable between the SI and control mice, suggesting that the enlargement of this particular fat depot 
was due to adipose hyperplasia rather than hypertrophy. Thus, the SI model may provide us a way to study the different mechanisms of adipose tissue expansion. The hypertrophy of adipocytes can also trigger a chronic low-grade inflammatory state as adipocytes become distressed and undergo necrosis/apoptosis, consequently recruiting macrophages $[18,56]$. In addition, as fat accumulates, adipokines and chemokines are secreted from adipocytes into the systemic circulation, attracting macrophages from the bloodstream into the expanding adipose tissue. In turn these resident macrophages release more cytokines (e.g., TNF- $\alpha$ and IL-6), which further stimulates the secretion of proinflammatory adipokines, resulting in a vicious cycle between adipocytes and macrophages inside the fat depot [57]. Given the different mechanisms of tissue expansion of the different WAT depots, it may be interesting to study the local inflammatory and immune response (cytokines) of the various WAT depots.

Although the present study focused on the WAT due to their direct relevance in obesity, the mechanism and effects of the enlargement of the BAT in stress-induced metabolic dysfunction warrant further study. BAT is the main site of nonshivering thermogenesis in mammals that dissipate energy through heat production. Three weeks of isolation was shown to increase both uncoupling protein-1 (UCP-1) content and sympathetic outflow in the BAT in rats [58]. Indeed, enhanced activation of BAT due to psychological stress has long been noted [59]. However, the role of this psychological stress-induced hyperthermia or "psychogenic fever" in stressrelated metabolic changes is unclear.

In contrast to the increased food intake observed by Sakakibara et al. [37], we found a reduction in food intake in the SI animals. A couple of notable differences between the two studies may contribute to this discrepancy. First, they used 4 week old mice instead of 12 week old adult mice. Secondly, they used reduced bedding to induce a more severe stress response. Taken together, our data suggest that increase in food intake was unlikely a primary cause for the increase in adiposity caused by isolation stress. The same group also reported changes in a lipid metabolism-related pathway in the liver of SI mice prior to body weight change [60]. Gene expression changes favoring the activation of the lipid biosynthesis pathway controlled by sterol regulatory element binding factor 1 (srebf1) would likely lead to the accumulation of fat. Another study reported an upregulation of metabolic genes in the mammary adipocytes of SI mice that coincided with increased lipid synthesis and leptin secretion [61]. Thus, changes in adipocyte gene expression and physiology are likely to result in the increase in adiposity. It is also possible that the increase in adiposity without body weight change was a result of differential energy partitioning, since SI can lead to reductions in bone mineral content and soft-lean tissue mass [38].

The metabolic chamber monitoring also revealed decreases in RER and heat produced in the SI mice, around times of light-dark transitions. On the other hand, activity level was comparable between single- and group-housed mice, although others have reported increased locomotion in socially isolated mice [62]. One factor to consider is the potentially greater stress in the group-housed mice when subjected to individual housing in the metabolic chamber, which may affect activity level. The reduced RER indicates that SI mice utilized more fatty acid as energy source compared to group-housed mice, which used more carbohydrate. This is consistent with the higher adiposity of the SI mice. Although the total heat produced was not significantly different between the two groups, the reduction in heat production during certain times of the day in the SI mice might contribute to a lower energy expenditure over time, contributing to an increase in adiposity in the SI mice.

\section{Conclusions}

In summary, here we show that long-term social isolation induced an increase in adiposity prior to any significant body weight gain. The expansion of adipose tissue was due to hyperplasia in the WATe and hypertrophy (with or without hyperplasia) in the WATi and WATr. These animals also exhibited blunted metabolic responses during light-dark transition that warrants further study.

\section{Conflict of Interests}

The authors declare that there is no conflict of interests regarding the publication of this paper.

\section{Acknowledgments}

The authors thank the Ohio State University Medical Center core facility for assistance with the WAT section preparation. Funding for this study was provided by the National Institute of Health (RO1NS044576).

\section{References}

[1] A. Berry, V. Bellisario, S. Capoccia et al., "Social deprivation stress is a triggering factor for the emergence of anxiety- and depression-like behaviours and leads to reduced brain BDNF levels in C57BL/6J mice," Psychoneuroendocrinology, vol. 37, no. 6, pp. 762-772, 2012.

[2] C. Ros-Simó and O. Valverde, "Early-life social experiences in mice affect emotional behaviour and hypothalamic-pituitaryadrenal axis function," Pharmacology Biochemistry and Behavior, vol. 102, no. 3, pp. 434-441, 2012.

[3] R. J. Katz, "Animal models and human depressive disorders," Neuroscience \& Biobehavioral Reviews, vol. 5, no. 2, pp. 231-246, 1981.

[4] A. Bartolomucci, P. Palanza, P. Sacerdote et al., "Individual housing induces altered immuno-endocrine responses to psychological stress in male mice," Psychoneuroendocrinology, vol. 28, no. 4, pp. 540-558, 2003.

[5] A. Guidotti, E. Dong, K. Matsumoto, G. Pinna, A. M. Rasmusson, and E. Costa, "The socially-isolated mouse: A model to study the putative role of allopregnanolone and $5 \alpha$ dihydroprogesterone in psychiatric disorders," Brain Research Reviews, vol. 37, no. 1-3, pp. 110-115, 2001.

[6] K. Nonogaki, K. Nozue, and Y. Oka, "Social isolation affects the development of obesity and type 2 diabetes in mice," Endocrinology, vol. 148, no. 10, pp. 4658-4666, 2007. 
[7] R. Sinha and A. M. Jastreboff, "Stress as a common risk factor for obesity and addiction," Biological Psychiatry, vol. 73, no. 9, pp. 827-835, 2013.

[8] A. W. Smith, A. Baum, and R. R. Wing, "Stress and weight gain in parents of cancer patients," International Journal of Obesity, vol. 29, no. 2, pp. 244-250, 2005.

[9] H. Kim, W.-W. Whang, H.-T. Kim et al., "Expression of neuropeptide $\mathrm{Y}$ and cholecystokinin in the rat brain by chronic mild stress," Brain Research, vol. 983, no. 1-2, pp. 201-208, 2003.

[10] S. C. Weninger, L. J. Muglia, L. Jacobson, and J. A. Majzoub, "CRH-deficient mice have a normal anorectic response to chronic stress," Regulatory Peptides, vol. 84, no. 1-3, pp. 69-74, 1999.

[11] S. Bhatnagar and C. Vining, "Facilitation of hypothalamicpituitary-adrenal responses to novel stress following repeated social stress using the resident/intruder paradigm," Hormones and Behavior, vol. 43, no. 1, pp. 158-165, 2003.

[12] R. S. Sherwin and L. Sacca, "Effect of epinephrine on glucose metabolism in humans: contribution of the liver," American Journal of Physiology-Endocrinology and Metabolism, vol. 10, no. 2, pp. E157-E165, 1984.

[13] S. C. Heinrichs, F. Menzaghi, E. M. Pich, R. L. Hauger, and G. F. Koob, "Corticotropin-releasing factor in the paraventricular nucleus modulates feeding induced by neuropeptide Y," Brain Research, vol. 611, no. 1, pp. 18-24, 1993.

[14] S. C. Heinrichs and D. Richard, "The role of corticotropinreleasing factor and urocortin in the modulation of ingestive behavior," Neuropeptides, vol. 33, no. 5, pp. 350-359, 1999.

[15] P. J. Currie, "Integration of hypothalamic feeding and metabolic signals: focus on neuropeptide Y," Appetite, vol. 41, no. 3, pp. 335-337, 2003.

[16] D. Richard, Q. Lin, and E. Timofeeva, "The corticotropinreleasing factor family of peptides and CRF receptors: their roles in the regulation of energy balance," European Journal of Pharmacology, vol. 440, no. 2-3, pp. 189-197, 2002.

[17] J. Maniam and M. J. Morris, "The link between stress and feeding behaviour," Neuropharmacology, vol. 63, no. 1, pp. 97110, 2012.

[18] I. Kyrou and C. Tsigos, "Stress hormones: physiological stress and regulation of metabolism," Current Opinion in Pharmacology, vol. 9, no. 6, pp. 787-793, 2009.

[19] T. W. Castonguay, "Glucocorticoids as modulators in the control of feeding," Brain Research Bulletin, vol. 27, no. 3-4, pp. 423-428, 1991.

[20] M. F. Dallman, N. C. Pecoraro, S. E. la Fleur et al., "Glucocorticoids, chronic stress, and obesity," Progress in Brain Research, vol. 153, pp. 75-105, 2006.

[21] M. F. Dallman, N. C. Pecoraro, and S. E. La Fleur, "Chronic stress and comfort foods: self-medication and abdominal obesity," Brain, Behavior, and Immunity, vol. 19, no. 4, pp. 275-280, 2005.

[22] M. E. Bell, S. Bhatnagar, J. Liang, L. Soriano, T. R. Nagy, and M. F. Dallman, "Voluntary sucrose ingestion, like corticosterone replacement, prevents the metabolic deficits of adrenalectomy," Journal of Neuroendocrinology, vol. 12, no. 5, pp. 461-470, 2000.

[23] S. Bhatnagar, M. E. Bell, J. Liang, L. Soriano, T. R. Nagy, and M. F. Dallman, "Corticosterone facilitates saccharin intake in adrenalectomized rats: does corticosterone increase stimulus salience?" Journal of Neuroendocrinology, vol. 12, no. 5, pp. 453460, 2000.
[24] M. F. Dallman, "Stress-induced obesity and the emotional nervous system," Trends in Endocrinology and Metabolism, vol. 21, no. 3, pp. 159-165, 2010.

[25] M. F. Dallman, N. Pecoraro, S. F. Akana et al., "Chronic stress and obesity: a new view of "comfort food"' Proceedings of the National Academy of Sciences of the United States of America, vol. 100, no. 20, pp. 11696-11701, 2003.

[26] E. Leigh Gibson, "Emotional influences on food choice: sensory, physiological and psychological pathways," Physiology and Behavior, vol. 89, no. 1, pp. 53-61, 2006.

[27] S. J. Torres and C. A. Nowson, "Relationship between stress, eating behavior, and obesity," Nutrition, vol. 23, no. 11-12, pp. 887-894, 2007.

[28] J. P. Block, Y. He, A. M. Zaslavsky, L. Ding, and J. Z. Ayanian, "Psychosocial stress and change in weight among US adults," The American Journal of Epidemiology, vol. 170, no. 2, pp. 181192, 2009.

[29] A. Serlachius, M. Hamer, and J. Wardle, "Stress and weight change in university students in the United Kingdom," Physiology and Behavior, vol. 92, no. 4, pp. 548-553, 2007.

[30] O. Martí, J. Martí, and A. Armario, "Effects of chronic stress on food intake in rats: influence of stressor intensity and duration of daily exposure," Physiology and Behavior, vol. 55, no. 4, pp. 747-753, 1994.

[31] E. Epel, S. Jimenez, K. Brownell, L. Stroud, C. Stoney, and R. Niaura, "Are stress eaters at risk for the metabolic syndrome?" Annals of the New York Academy of Sciences, vol. 1032, pp. 208210, 2004.

[32] A. A. Stone and K. D. Brownell, "The stress-eating paradox: multiple daily measurements in adult males and females," Psychology \& Health, vol. 9, no. 6, pp. 425-436, 1994.

[33] L. E. Kuo, M. Czarnecka, J. B. Kitlinska, J. U. Tilan, R. Kvetňanský, and Z. Zukowska, "Chronic stress, combined with a high-fat/high-sugar diet, shifts sympathetic signaling toward neuropeptide $\mathrm{Y}$ and leads to obesity and the metabolic syndrome," Annals of the New York Academy of Sciences, vol. 1148, pp. 232-237, 2008.

[34] C. Dodt, P. Lönnroth, J. P. Wellhöner, H. L. Fehm, and M. Elam, "Sympathetic control of white adipose tissue in lean and obese humans," Acta Physiologica Scandinavica, vol. 177, no. 3, pp. 351357, 2003.

[35] L. Sominsky and S. J. Spencer, "Eating behavior and stress: a pathway to obesity," Frontiers in Psychology, vol. 5, p. 434, 2014.

[36] T. I. Takemoto, T. Suzuki, and T. Miyama, "Effects of isolation on mice in relation to age and sex," The Tohoku Journal of Experimental Medicine, vol. 117, no. 2, pp. 153-165, 1975.

[37] H. Sakakibara, A. Suzuki, A. Kobayashi et al., "Social isolation stress induces hepatic hypertrophy in C57BL/6J mice," Journal of Toxicological Sciences, vol. 37, no. 5, pp. 1071-1076, 2012.

[38] T. R. Nagy, D. Krzywanski, J. Li, S. Meleth, and R. Damond, "Effect of group vs. single housing on phenotypic variance in C57BL/6J mice," Obesity Research, vol. 10, no. 5, pp. 412-415, 2002.

[39] M. Guo, C. F. Wu, W. Liu, J. Y. Yang, and D. Chen, "Sex difference in psychological behavior changes induced by long-term social isolation in mice," Progress in Neuro-Psychopharmacology and Biological Psychiatry, vol. 28, no. 1, pp. 115-121, 2004.

[40] T. Kaji and K. Nonogaki, "Contribution of central SGK-1 to the acute phase responses of mice to social isolation," Frontiers in Bioscience-Elite, vol. 2, no. 4, pp. 1355-1361, 2010. 
[41] K. Yamada, H. Ohki-Hamazaki, and K. Wada, "Differential effects of social isolation upon body weight, food consumption, and responsiveness to novel and social environment in bombesin receptor subtype-3 (BRS-3) deficient mice," Physiology \& Behavior, vol. 68, no. 4, pp. 555-561, 2000.

[42] A. Bartolomucci, A. Cabassi, P. Govoni et al., "Metabolic consequences and vulnerability to diet-induced obesity in male mice under chronic social stress," PLoS ONE, vol. 4, no. 1, Article ID e4331, 2009.

[43] Y. Arita, S. Kihara, N. Ouchi et al., "Paradoxical decrease of an adipose-specific protein, adiponectin, in obesity," Biochemical and Biophysical Research Communications, vol. 257, no. 1, pp. 79-83, 1999.

[44] L. Cao, E.-J. D. Lin, M. C. Cahill, C. Wang, X. Liu, and M. J. During, "Molecular therapy of obesity and diabetes by a physiological autoregulatory approach," Nature Medicine, vol. 15, no. 4, pp. 447-454, 2009.

[45] L. Cao, X. Liu, E.-J. D. Lin et al., "Environmental and genetic activation of a brain-adipocyte BDNF/leptin axis causes cancer remission and inhibition," Cell, vol. 142, no. 1, pp. 52-64, 2010.

[46] M. H. Rasmussen, "Obesity, growth hormone and weight loss," Molecular and Cellular Endocrinology, vol. 316, no. 2, pp. 147153, 2010.

[47] J. Frystyk, C. Skjaerbaek, E. Vestbo, S. Fisker, and H. Orskov, "Circulating levels of free insulin-like growth factors in obese subjects: the impact of type 2 diabetes," Diabetes/Metabolism Research and Reviews, vol. 15, no. 5, pp. 314-322, 1999.

[48] S. Y. Nam, E. J. Lee, K. R. Kim et al., "Effect of obesity on total and free insulin-like growth factor (IGF)-1, and their relationship to IGF-binding protein (BP)-1, IGFBP-2, IGFBP-3, insulin, and growth hormone," International Journal of Obesity, vol. 21, no. 5, pp. 355-359, 1997.

[49] J. M. Gómez, F. J. Maravall, N. Gómez, M. Á. Navarro, R. Casamitjana, and J. Soler, "The IGF-I system component concentrations that decrease with ageing are lower in obesity in relationship to body mass index and body fat," Growth Hormone and IGF Research, vol. 14, no. 2, pp. 91-96, 2004.

[50] M. H. Rasmussen, J. Frystyk, T. Andersen, L. Breum, J. S. Christiansen, and J. Hilsted, "The impact of obesity, fat distribution, and energy restriction on insulin-like growth factor-1 (IGF-1), IGF-binding protein-3, insulin, and growth hormone," Metabolism: Clinical and Experimental, vol. 43, no. 3, pp. 315319, 1994.

[51] E. S. Epel, "Psychological and metabolic stress: a recipe for accelerated cellular aging?" Hormones, vol. 8, no. 1, pp. 7-22, 2009.

[52] E. Bernton, D. Hoover, R. Galloway, and K. Popp, "Adaptation to chronic stress in military trainees. Adrenal androgens, testosterone, glucocorticoids, IGF-1, and immune function," Annals of the New York Academy of Sciences, vol. 774, pp. 217231, 1995.

[53] N. Uschold-Schmidt, K. D. Nyuyki, A. M. Füchsl, I. D. Neumann, and S. O. Reber, "Chronic psychosocial stress results in sensitization of the HPA axis to acute heterotypic stressors despite a reduction of adrenal in vitro ACTH responsiveness," Psychoneuroendocrinology, vol. 37, no. 10, pp. 1676-1687, 2012.

[54] S. O. Reber, L. Birkeneder, A. H. Veenema et al., "Adrenal insufficiency and colonic inflammation after a novel chronic psychosocial stress paradigm in mice: Implications and mechanisms," Endocrinology, vol. 148, no. 2, pp. 670-682, 2007.

[55] E. J. Lin, M. Sun, E. Y. Choi, D. Magee, C. W. Stets, and M. J. During, "Social overcrowding as a chronic stress model that increases adiposity in mice," Psychoneuroendocrinology, vol. 51, pp. 318-330, 2015.

[56] B. O. V. Shum, C. R. Mackay, C. Z. Gorgun et al., “The adipocyte fatty acid-binding protein $\mathrm{aP} 2$ is required in allergic airway inflammation," The Journal of Clinical Investigation, vol. 116, no. 8, pp. 2183-2192, 2006.

[57] K. E. Wellen and G. S. Hotamisligil, "Obesity-induced inflammatory changes in adipose tissue," Journal of Clinical Investigation, vol. 112, no. 12, pp. 1785-1788, 2003.

[58] G. Cvijic, I. Lakic, P. Vujovic et al., "Single and combined effects of acute and chronic non-thermal stressors on rat interscapular brown adipose tissue metabolic activity," Archives of Biological Science Belgrade, vol. 65, no. 3, pp. 919-927, 2013.

[59] T. Oka, K. Oka, and T. Hori, "Mechanisms and mediators of psychological stress-induced rise in core temperature," Psychosomatic Medicine, vol. 63, no. 3, pp. 476-486, 2001.

[60] K. Motoyama, Y. Nakai, T. Miyashita et al., "Isolation stress for 30 days alters hepatic gene expression profiles, especially with reference to lipid metabolism in mice," Physiological Genomics, vol. 37, no. 2, pp. 79-87, 2009.

[61] P. A. Volden, E. L. Wonder, M. N. Skor et al., "Chronic social isolation is associated with metabolic gene expression changes specific to mammary adipose tissue," Cancer Prevention Research, vol. 6, no. 7, pp. 634-645, 2013.

[62] L. A. Hilakivi, M. Ota, and R. G. Lister, "Effect of isolation on brain monoamines and the behavior of mice in tests of exploration, locomotion, anxiety and behavioral "despair"', Pharmacology Biochemistry and Behavior, vol. 33, no. 2, pp. 371374, 1989. 


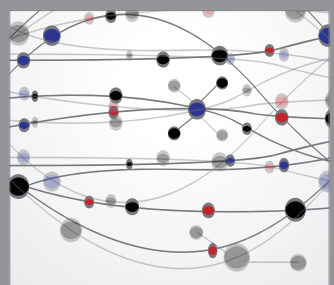

The Scientific World Journal
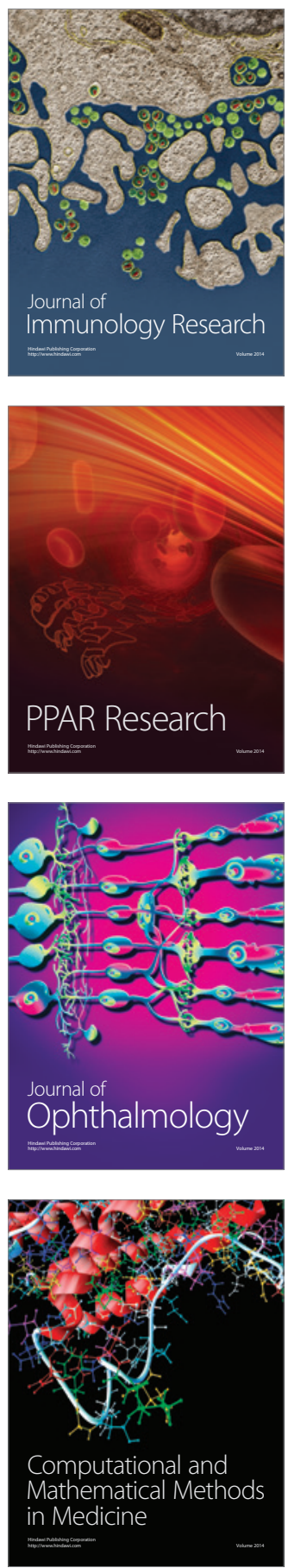

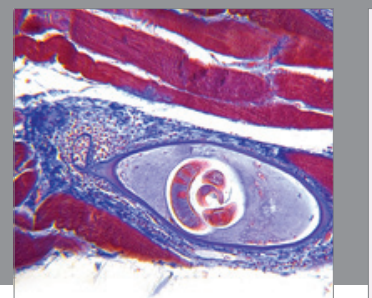

Gastroenterology

Research and Practice
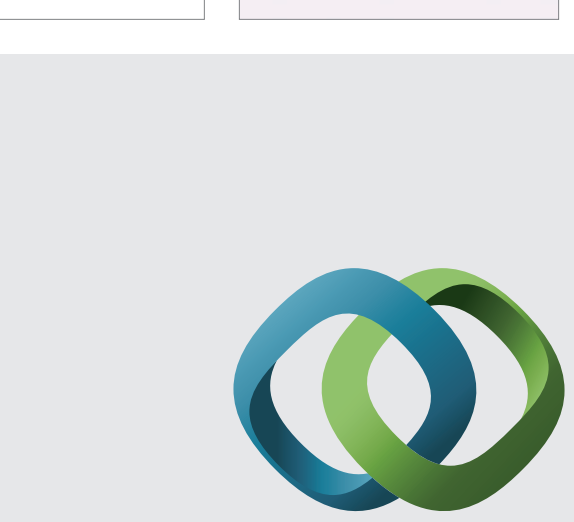

\section{Hindawi}

Submit your manuscripts at

http://www.hindawi.com
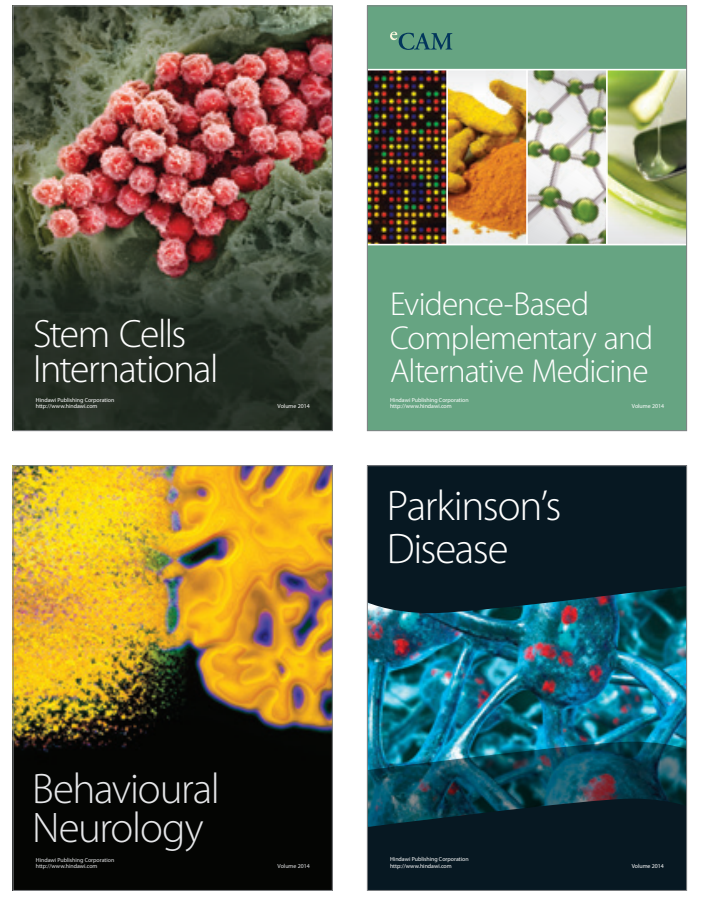
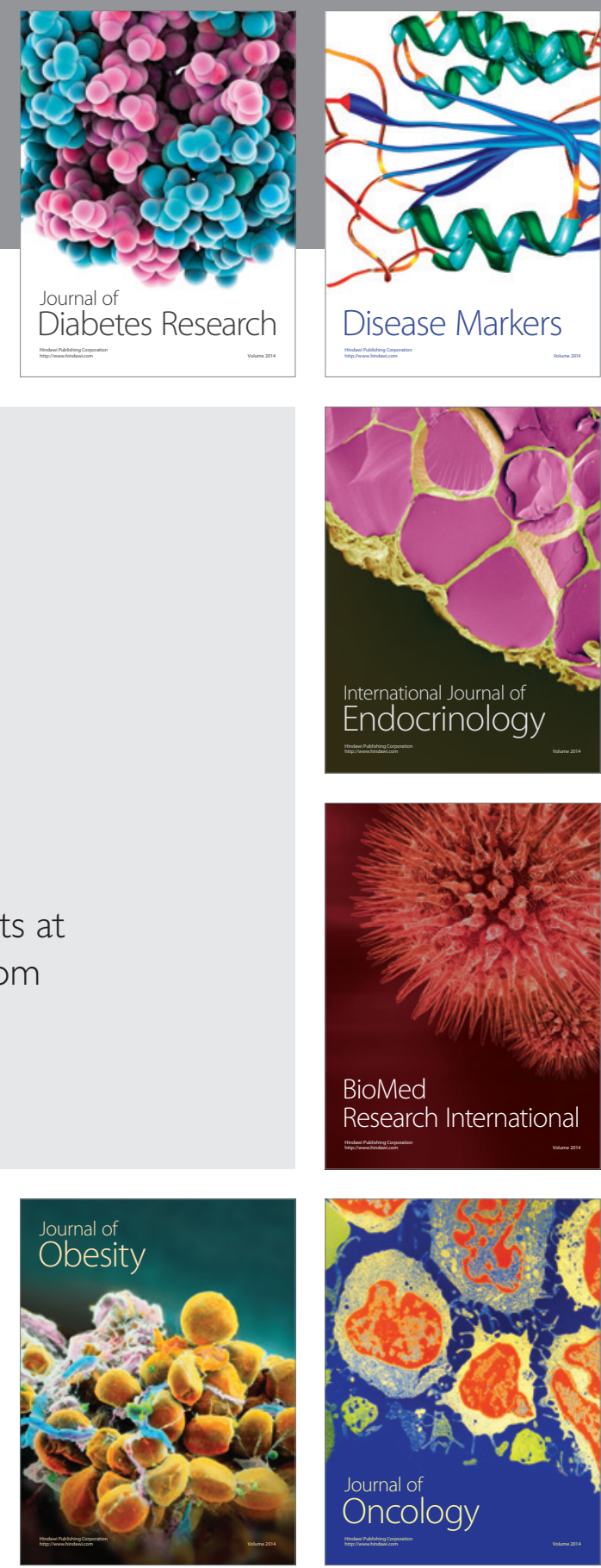

Disease Markers
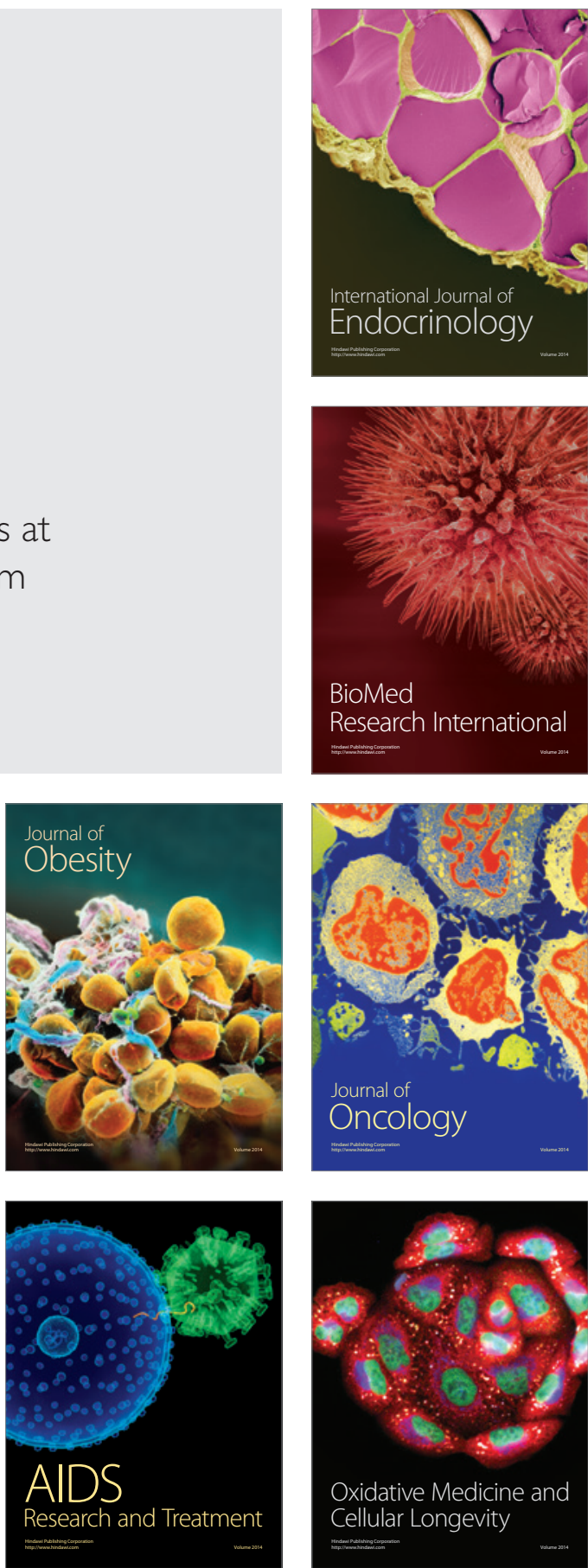